\title{
Comportamiento sexual en los pacientes de hemodiálisis en Melilla
}

Victoria Eugenia Muñoz García* - Ana María Vaca Ruíz* - Esperanza Romero Estudillo** Juana García Criado* - Aziza Bagdad Abselam* - Nayet Mohamed Maanan*

*DUE. Hospital Comarcal de Melilla

** DUE. LCDA. Enfermera EAP. Profesora de la EUE. Melilla

\section{Resumen}

Los trastornos de la disfunción sexual no son infrecuentes en las personas con Insuficiencia Renal Crónica Terminal en diálisis. Nuestro objetivo fue conocer el comportamiento sexual en los pacientes de Hemodiálisis en Melilla, y saber si padecen alguna alteración o disfunción sexual.

Se trata de estudio descriptivo transversal en una muestra de 38 pacientes. Se recogen los datos en un cuestionario confeccionado para tal fin que se sometió a un juicio de expertos y se tradujo al árabe por la barrera idiomática. Los resultados muestran que el $57.89 \%$ son mujeres y el $42.10 \%$ varones. La edad media oscila entre los 40 en varones y los 70 años en mujeres. En el $56.25 \%$ de lo varones y el $31.8 \%$ de las mujeres han disminuido el deseo sexual desde que están en Hemodiálisis. La disminución de la libido afecta más a las mujeres que a los varones. Estarían dispuestos a tratar la disfunción sexual el $75 \%$ de los hombres y el $37.5 \%$ relacionan el factor psicológico como causante.

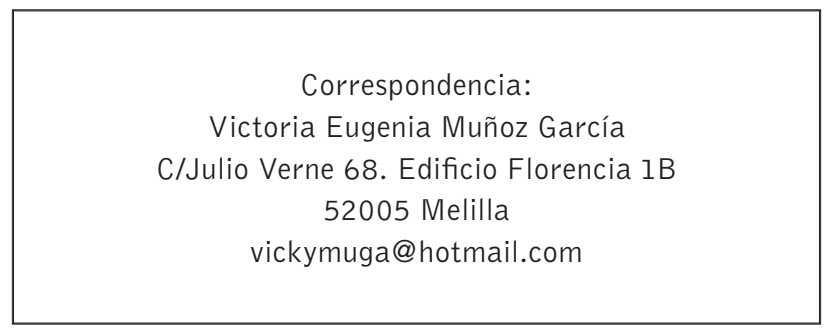

\section{PALABRAS CLAVE: \\ - SEXUALIDAD \\ - DISFUNCIÓN SEXUAL \\ - LIBIDO \\ - HEMODIÁLISIS}

\section{Sexual behaviour in haemodialysis patients in Melilla}

\section{Abstract}

Sexual dysfunction disorders are not infrequent in people with Terminal Chronic Renal Insufficiency undergoing dialysis. Our aim was to find out the sexual behaviour in haemodialysis patients in Melilla, and determine whether they suffered any sexual alteration or dysfunction.

This is a transversal descriptive study carried out on a sample of 38 patients. The data were compiled on a questionnaire prepared for this purpose which was submitted to the opinion of experts and was tranlated into Arabic due to the language barrier. The results show that $57.89 \%$ are women and $42.10 \%$ are men. The average age is around 40 in men and 70 in women. Sex drive has fallen in $56.25 \%$ of the men and $31.8 \%$ of the women since they have been on haemodialysis. The reduction in libido affects women more than men. $75 \%$ of the men would be willing to treat the sexual dysfunction and $37.5 \%$ list the psychological factor as the cause.

KEY WORDS:

- SEXUALITY

- SEXUAL DYSFUNCTION

- LIBIDO

- HAEMODIALYSIS 


\section{Introducción}

La relación entre sexualidad y salud es tal que un grupo de expertos de la Organización Panamericana de la Salud y la Organización Mundial de la Salud (OMS) ${ }^{1}$, en colaboración con la Asociación Mundial de Sexología, propuso entre otras, la siguiente definición: "la salud sexual es la experiencia del proceso permanente de consecución de bienestar físico, psicológico y sociocultural relacionado con la sexualidad".

Existe evidencia y unanimidad científica en atribuir como principal causa de la pérdida de la salud a las disfunciones sexuales; citamos entre otras, la ausencia o disminución del deseo sexual, la disfunción orgásmica, la dispareunia, el vaginismo, la disfunción eréctil, la eyaculación precoz, etc. La disfunción sexual es un síntoma altamente prevalente en los pacientes con insuficiencia renal crónica y el origen de la misma todavía no es bien conocido aunque puede ser causada por la interacción de una serie de factores como el estado urémico, el afrontamiento ineficaz a la diálisis, una percepción disminuida de bienestar físico, alteraciones hormonales y las patologías asociadas (diabetes, enfermedad vascular, neuropatía urémica, depresión...), y/o al efecto secundario de los fármacos ${ }^{2}$. Hay que tener en cuenta que a veces aunque haya una causa orgánica que pueda ser causante de la disfunción, se suelen añadir otros factores psicológicos que hacen más complejo el cuadro general; las causas psicológicas más habituales son el estrés, la ansiedad, sentimientos de culpa, de inferioridad o baja autoestima y el temor a "no poder" en el momento del acto sexual, entre otras ${ }^{3}$.

Es evidente por otra parte, que los pacientes con Insuficiencia Renal Crónica Terminal (IRCT) en tratamiento sustitutivo sufren una serie de cambios fisiológicos y psicológicos que influyen de manera importante en su calidad de vida. Además la insuficiencia renal, como hemos mencionado anteriormente, cursa con otras patologías asociadas que ya padecían antes, 0 bien, han aparecido después. Así, la diabetes mellitus, la hipertensión arterial, la cardiopatía isquémica, la anemia, alteraciones hormonales, etc., son algunos de los problemas de salud que pueden agudizar las disfunciones sexuales. Diversos estudios ponen de manifiesto una elevada prevalencia de disfunción sexual entre los pacientes portadores de una IRCT. Así, cabe mencionar los trastornos hormonales en los hombres que están sometidos a tratamiento sustitutivo renal con atrofia testicular, disminución de la espermatogénesis y descenso de los niveles de testosterona y la presentación de impotencia parcial o total. En las mujeres puede haber descenso de estrógenos, amenorrea 0 hipomenorrea e incluso puede haber una disminución del tejido mamario, menor lubricación vaginal, vaginitis atrófica e infertilidad. La mayoría de ellas presentan alguna disfunción sexual, algunas pueden experimentar dispareunia debida al déficit de estrógenos y la sequedad vaginal resultante, pero también podría deberse a la hiperprolactinemia, la disfunción gonadal, la depresión, el hiperparatiroidismo, el déficit de cinc y el cambio de la imagen corporal.

A pesar del bienestar físico que produce el tratamiento sustitutivo renal en los pacientes en programa de $\mathrm{He}-$ modiálisis (HD), la disfunción sexual se hace evidente en la fase urémica, empeorando una vez iniciada la diálisis que se manifiesta fundamentalmente como impotencia y disminución de la libido en el varón, y, por disminución del deseo sexual y defecto de lubricación en las mujeres, mientras que los pacientes inactivos sexualmente parecen tener una adaptación psicológica y emocional satisfactoria al problema, los pacientes que refieren una mejor condición sexual perciben también una mayor Calidad de Vida Relacionada con la Salud (CVRS) que se manifiesta en la realización de actividades sociales y cotidianas, en una vivencia emocional menos negativa, un apoyo social percibido algo mejor, y una percepción de que las cosas van razonablemente bien según Martín Díaz y col. (2006) ${ }^{4}$.

Además de los problemas físicos, los pacientes en diálisis se encuentran expuestos a varios tipos de estrés psicológico como la dependencia del procedimiento, las exigencias del cumplimiento de un régimen dietético y la toma de medicación; las pérdidas varias que sufren (trabajo, libertad, esperanza de vida, etc.), y la disfunción sexual asociada. La respuesta psicológica de cada paciente dependerá de su personalidad, del grado de apoyo familiar y social y del curso de la enfermedad. La complicación psicológica más frecuente en los pacientes en diálisis es la depresión que produce síntomas como trastornos del sueño, falta de apetito y una disminución del interés y las capacidades sexuales. La irritabilidad y la ansiedad son también frecuentes.

El objetivo del presente estudio es conocer el comportamiento sexual, $\mathrm{y}$, si los pacientes en programa de Hemodiálisis (HD) de la Unidad Dr. Antonio Torralbo 
Rigual de Melilla, sufren alguna alteración o disfunción sexual. Con la intención de dar una mejor respuesta al problema general surgen los siguientes problemas específicos que queremos analizar:

1. ¿Hay diferencias en el comportamiento sexual entre hombres y mujeres en programa de HD de Melilla?

2. ¿Existe alguna relación causa-efecto en relación al tiempo de permanencia de terapia HD y la disfunción sexual, en el caso de que la hubiere?

3. ¿Existen diferencias significativas entre los factores orgánicos y psicológicos? ¿Qué actitudes presentan los pacientes ante la disfunción sexual?

\section{Material y método}

\section{Población y muestra}

La unidad de HD de Melilla cuenta con una población de 66 pacientes en el programa, la muestra que accede y se somete al estudio corresponde a 40 personas, previo consentimiento y asegurándoles la confidencialidad de los datos. Se han excluido a 2 personas por no contestar el $50 \%$ de las preguntas, resultando una muestra de 38 personas (22 mujeres y 16 varones). La edad oscila entre los 18 y 80 años; el tiempo de permanencia en el programa de HD es de 6 meses a 5 años. 0tras variables a tener en cuenta son el estado civil, el nivel de formación académica y las practicas religiosas 0 morales. Es un estudio descriptivo transversal.

\section{Instrumentos de medición y Procedimiento de recogida de datos:}

1. Cuestionario. Se ha confeccionado un cuestionario (anexo 1) con un total de 24 ítems que se ha sometido a un juicio de expertos (nefrólogos, enfermeras y psicólogos); además, se ha realizado una versión del cuestionario en árabe (figura 1) debido a la barrera idiomática, ya que, Melilla es una ciudad fronteriza situada en el norte de África, con una gran diversidad cultural por la convivencia de culturas diferentes, siendo mayoritarias las etnias berebere y europea.

2. Analíticas de FSH (testosterona) y LH (prolactina). Se someten a analítica hormonal el $34.84 \%$ de los pacientes (el $47.8 \%$ son varones y el $52.1 \%$ mujeres).
3. Criterios de inclusión. Tener una edad comprendida entre los 18 y 80 años y aceptar voluntariamente contestar al cuestionario al menos en un $80 \%$ de las preguntas. Se identifican como nulos 2 cuestionarios por falta de respuestas al mismo.

4. Temporalización del estudio. El trabajo de recogida de datos se inicia en el mes de abril y finalizó en mayo de 2009.

El análisis de datos se procesó mediante el paquete estadístico SPSS para Windows en su versión 15.0.

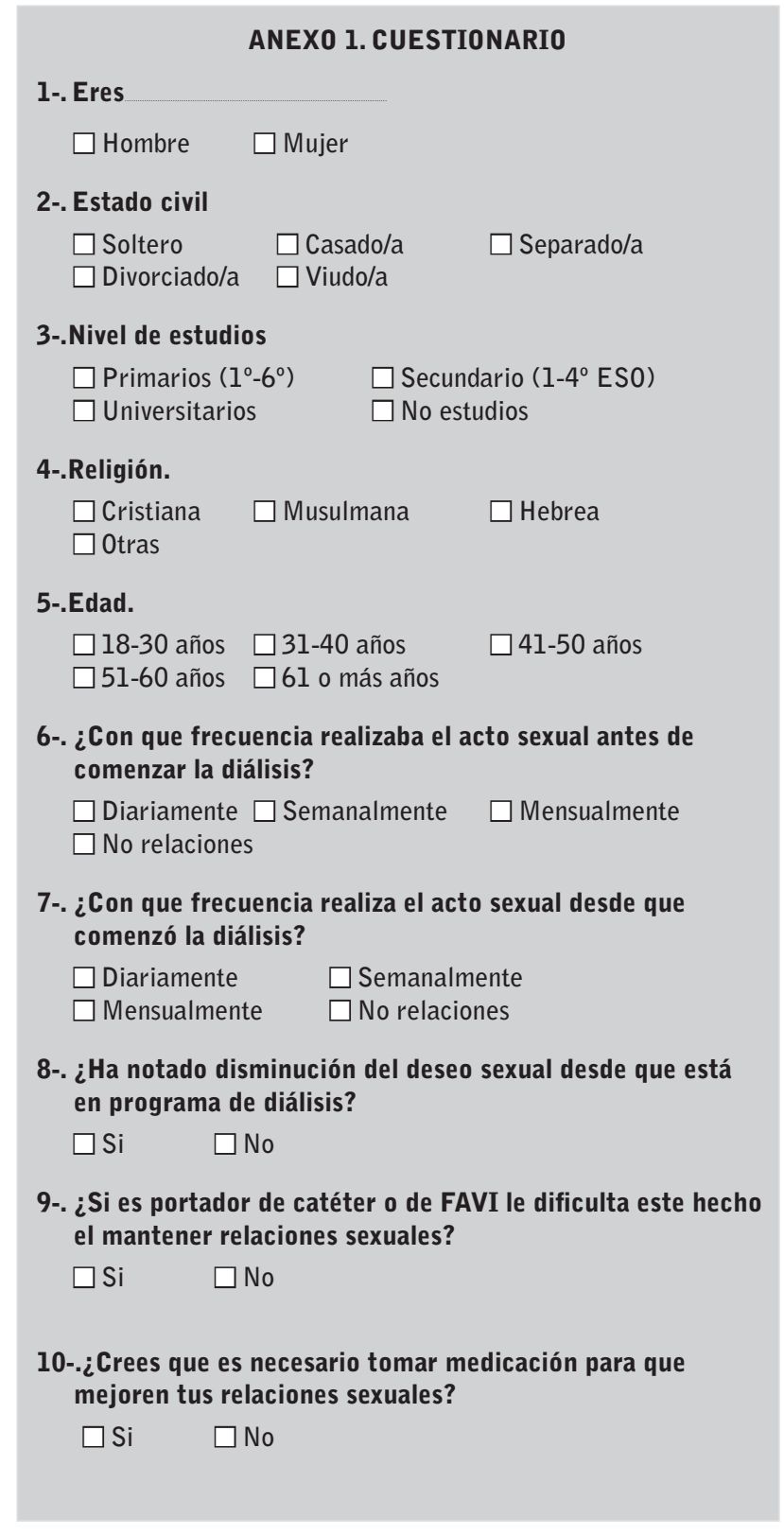


11-. ¿Cuánto tiempo lleva en hemodiálisis?
$\square$ Menos de 6 meses
$\square 6$ meses -2 años
$\square 3-5$ años
$\square$ Más de 5 años

12-. ¿Con qué edad comenzó a dializarse?
$\square 18-30$ años
$\square 31-40$ años
$\square$ 41-50 años
$\square 51-60$ años
$\square 61$ o más años

13-. ¿Ha cambiado sus hábitos sexuales en función de su turno del programa de hemodiálisis?

$\square$ Si $\square$ No

14-. ¿Es usted diabético?
$\square \mathrm{Si}$
$\square$ No

15-. ¿Toma usted tratamiento para la tensión?

$\square$ Si $\square$ No

16-. ¿Ha sufrido alguna vez un infarto?

$\square \mathrm{Si} \quad \square$ No

17-. ¿Se considera usted menos atractivo sexualmente por el hecho de estar en hemodiálisis?

$\square \mathrm{Si} \quad \square$ No

18-. ¿Considera que puede ser rechazado por su pareja por el hecho de estar en hemodiálisis?

$\square \mathrm{Si} \quad \square$ No

19-. ¿Ha tratado de buscar información vía Internet, folletos, libros, asociaciones acerca de la conducta sexual en hemodiálisis?

$\square \mathrm{Si} \quad \square$ No

20-. ¿Afecta a sus relaciones sexuales el hecho de estar o no estar en la lista de transplantes?

$\square$ Sí afecta $\quad \square$ No afecta

$\square$ No estoy en la lista de transplantes $\square$ No sabe/no contesta

21-. ¿Usa medios anticonceptivos desde que esta sometido a tratamiento de hemodiálisis?

$\square \mathrm{Si} \quad \square$ No

22-. ¿Ha estado sometido a tratamiento de fertilidad durante el tiempo que lleva en hemodiálisis?

$\square \mathrm{Si} \quad \square$ No

23-. ¿Ha tenido hijos/as durante el tiempo que lleva sometido a tratamiento de hemodiálisis?

$\square \mathrm{Si} \quad \square$ No

24-. ¿Ha tratado de tener hijos durante el tiempo que lleva en hemodiálisis?

$\square \mathrm{Si} \quad \square$ No

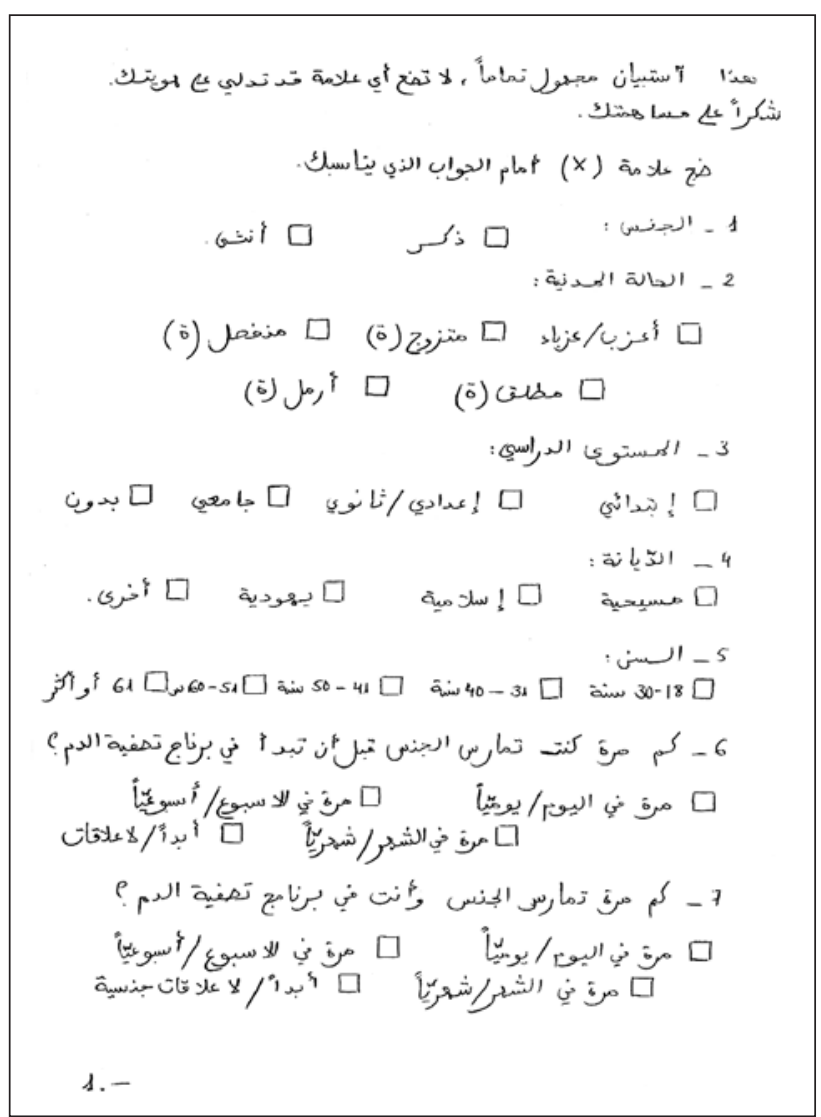

Figura 1. Cuestionario en árabe

\section{Resultados}

Se han sometido al estudio el $57.57 \%$ de los pacientes en programa de HD y de estos el $57.89 \%$ son mujeres y el $42.10 \%$ varones. La edad media de los encuestados oscila entre los 40 años en varones y los 70 años en mujeres. Son de la etnia bereber el $65.7 \%$ de los pacientes y de la etnia europea el $34.2 \%$. Poseen estudios académicos universitarios el $9.09 \%$ de las mujeres bereberes y el $12.5 \%$ de los varones europeos; el $45.45 \%$ de las mujeres de etnia bereber son analfabetas frente a la mayoría de la mujeres europeas que en un $22.72 \%$ tienen estudios primarios y ninguna de estas estudios universitarios; así mismo, el $18.7 \%$ de los hombres bereberes se benefician de estudios secundarios, coincidiendo con la misma proporción de analfabetos en este grupo cultural. Presentan barrera idiomática importante el $13.15 \%$ de los pacientes, para los cuales se ha confeccionado el cuestionario en árabe.

El tiempo de permanencia en el programa de menos de 6 meses corresponde al $10.5 \%$ de los usuarios; el $36.8 \%$ llevan en el programa entre 6 meses y 2 años; 
de 3 a 5 años el $36.8 \%$ y un $15.7 \%$ de los pacientes permanecen más de 5 años en el programa.

El acceso para HD con fístula arteriovenosa interna (FAVI) corresponde al $57.8 \%$, y, son portadores de catéter permanente el $42.1 \%$ de los pacientes. Las enfermedades orgánicas que cursan como asociadas y que guardan relación en mayor o menor medida con la enfermedad renal, son en primer lugar, la HTA (71.05\%), seguido, de la DM $(63.15 \%)$. Hacen uso y tiene adherencia al tratamiento farmacológico antihipertensivo el $100 \%$ de los afectados.

A la pregunta si han notado diferencia en relación al deseo sexual desde que se iniciaron en el programa dialítico con respecto a antes de ser diagnosticados, han respondido los varones afirmativamente en el $56.25 \%$ y las mujeres en el $31.8 \%$. La disminución de la libido afecta más a las mujeres que a los varones, repercutiendo en una ausencia de la actividad sexual en el $68.18 \%$ de las encuestadas, mientras que antes de entrar el programa de HD, la ausencia de actividad sexual se representa en un $36 \%$. No utilizan ningún método anticonceptivo el $93.7 \%$ de los varones frente al $72.72 \%$ de las mujeres. Estarían dispuestos a tratar la disfunción sexual el 75\% de los hombres y el $22.72 \%$ de las mujeres.

Perciben el factor psicológico (alteración de la imagen corporal/rechazo de la pareja) como desencadenante de la disfunción sexual el $37.5 \%$ de los varones y el $31.8 \%$ de las mujeres (figura 2 ).

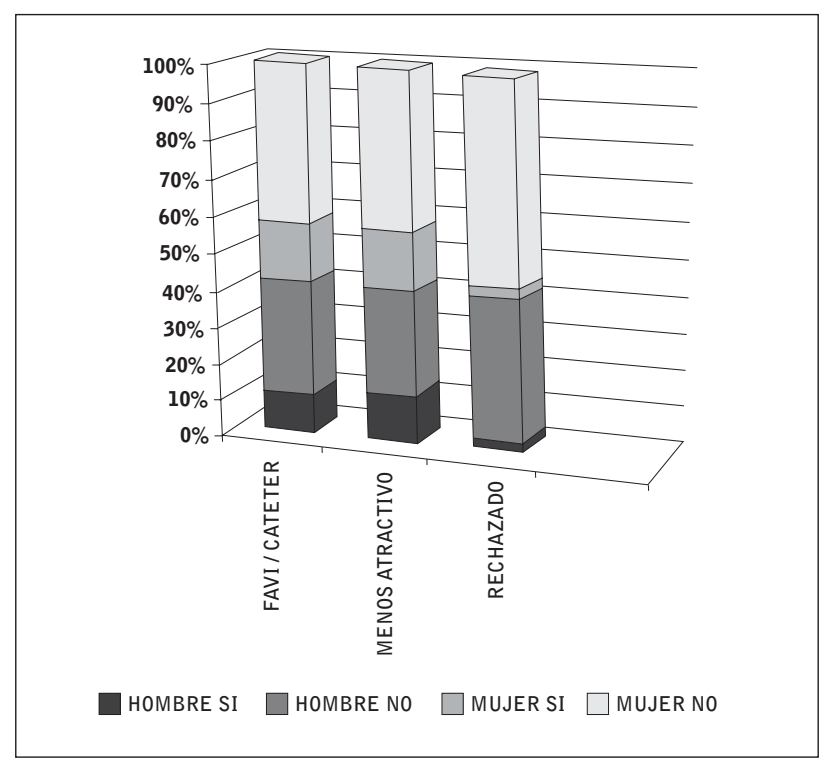

Figura 2. Factores psicológicos en hombres y mujeres que afectan a la actividad sexual

\section{Discusión}

Las disfunciones sexuales se consideran un tema sanitario de primer orden, porque pueden producir un deterioro potencial de la calidad de vida y del estado general de salud; así, Fernández $(2002)^{5}$ manifiesta que el $75 \%$ de los pacientes en HD padecen Disfunción Eréctil (DE); este motivo podría ser, entre otros, lo que últimamente ha incrementado las publicaciones sobre este tema. Asimismo, este dato revela que la conducta sexual de los pacientes en programa de HD de Melilla, sigue un patrón similar de afectación sexual que el resto de la población, ya que el $88 \%$ de los pacientes melillenses encuestados, así lo expresan. Desde la perspectiva de género, son las mujeres las que declaran en mayor proporción la disminución de la libido, llegando casi a doblar la cifra con respecto a antes de precisar el tratamiento sustitutivo; así, estas afirmaciones quedan recogidas por las autoras Andreu y Force $(2001)^{6}$ al manifestar un agravamiento de las alteraciones sexuales en las mujeres en tratamiento de HD con pérdida de la libido, alteraciones menstruales y disminución de la fertilidad. Por otra parte, en nuestro estudio, el varón esta más dispuesto a tratarse la disfunción sexual que la mujer, pero si tenemos en cuenta la edad de los encuestados -la edad media de las mujeres es de 70 años y la de los varones 40- y, que el envejecimiento en sí no frena la actividad sexual sino que son los problemas de salud los principales inconvenientes ${ }^{7}$, explicaría que la falta de deseo sexual en las mujeres está en relación con los problemas de salud, más que con la edad; encontramos en este grupo- mujeres- más elevada la proporción de HTA y DM, que junto con, los efectos secundarios de los antihipertensivos, demuestran, entre otras, tres causas importantes para las disfunciones sexuales ${ }^{2,4,5}$. Por otra parte, y en relación con la variable edad, encontramos en la bibliografía que las mujeres menopáusicas experimentan una reducción de su capacidad sexual y que el $20 \%$ de estas pacientes ven alteradas gravemente sus relaciones emocionales. Sin embargo, sólo el 18\% de las mujeres menopáusicas solicita ayuda médica y tarda unos 6 años en realizar la consulta, según se manifestó en el Congreso de la Sociedad Española de Ginecología y Obstetricia (SEG0, 2009)8. Lo que apuntaría a un comportamiento similar en nuestras pacientes. 


\section{Conclusiones}

1) Los pacientes en programa de HD de la Unidad de Melilla presentan un comportamiento sexual alterado junto con otros problemas de salud física como HTA y DM. Existen diferencias significativas en cuanto al género, edad y factores desencadenantes de las disfunciones sexuales.

2) Los varones reconocen en mayor medida haber sufrido un deterioro en el comportamiento sexual desde que iniciaron el tratamiento sustitutivo, pero las mujeres expresan una pérdida de la libido más importante que los varones.

3) Los varones presentan una actitud más positiva frente a la sexualidad, expresando su deseo de someterse a tratamiento. Sienten más el rechazo de sus parejas sexuales y lo identifican como factor psicológico desencadenante de la disfunción sexual.

4) Las mujeres llevan más tiempo en el programa de HD y son más mayores que los varones; están más afectadas por las enfermedades crónicas como la HTA, la DM y la menopausia; presentan una actitud más negativa que los hombres frente a la sexualidad relacionado con los problemas de salud físicos y no con la edad, ni con factores psicológicos. La disminución del deseo sexual les afectaba antes de entrar en el programa de HD.

5) Es recomendable seguir con estudios de investigación sobre la sexualidad y planificar intervenciones individuales que mejoren la calidad de vida de nuestros pacientes. Mantener unas relaciones afectivas satisfactorias y plenas, influye en el estado de ánimo de las personas e incluso puede actuar de forma favorable para prevenir sentimientos de tristeza, soledad e incluso, depresiones.

\section{Bibliografía}

1. Actas de una reunión de consulta convocada por la Organización Mundial de la Salud-Organización Panamericana de la Salud (OPS-OMS) en cola- boración con la Asociación Mundial de Sexología (WAS). Promoción de la salud sexual. Recomendaciones para la acción, (58 pp). Antigua Guatemala, Guatemala del 19 al 22 de mayo de 2000. Disponible en: http://www.paho.org/Spanish/AD/FCH/ AI/salud_sexual.pdf. (Consultado el 28 de mayo 2009).

2. Alcoba Valls S, García-Giralda Ruiz L, San Martin Blanco C. Guía de buenas prácticas clínicas en disfunciones sexuales. Madrid: Ministerio de Sanidad y Consumo; 2004. Disponible en: www.comsegovia. $\mathrm{com} / \mathrm{pdf} / \mathrm{guias} / \mathrm{GBPC} \% 20$ DISFUNCIONES\%20 SEXUALES.pdf\#page $=46$. (Consultado 4 de marzo de 2009).

3. Sánchez Bravo C, Carreño Meléndez J, Martínez Ramírez S, Gómez López ME. Disfunciones sexuales femeninas y masculinas: comparación de género en una muestra de la Ciudad de México. Salud Mental 2005; 28 (4).

4. Martín-Díaz F, Reig-Ferrer A, Ferrer-Cascales R. Función sexual y calidad de vida en pacientes varones de hemodiálisis. Nefrología 2006; 26 (4):452-460.

5. Fernández Vega F. Disfunción eréctil en el paciente hipertenso y renal. Nefrología 2002; Vol. XXII. Suplemento 2: 18-19.

6. Andreu Periz D, Force Sanmartín E. 500 cuestiones que plantea el cuidado del enfermo renal. $2^{\mathrm{a}}$ ed. Elsevier Masson; 2001.

7. Mosquera A. Los problemas de salud afectan más a la actividad sexual de las personas mayores que la edad que éstos tienen. "Imagínate que puedes". Congreso Nacional de la Sociedad Española de Médicos de Residencias (SEMER). Bilbao; 2007.

8. Martín Malavé $F$, Palacios $S$, Callejo J, Guinot $M$, Baró F, Rodríguez Bueno E, et al. La menopausia al inicio del siglo XXI. Congreso de la Sociedad Española de Ginecología y Obstetricia (SEGO), Barcelona; 2009. http://www.noticiasmedicas.es/. Consultado el 19 de agosto de 2009. 